\title{
Study on the particle shape of soft clay in Tianjin port
}

\author{
Xuekui Wang ${ }^{1,2 *}$, Dongjun $\mathrm{Li}^{1,2}$, Yaoting $\mathrm{Zhu}^{1,2}$ \\ ${ }^{1}$ CCCC Tianjin Port Engineering Institute Co., Ltd., Tianjin, 300222, China. \\ ${ }^{2}$ CCCC First Habor Engineering Co., Ltd., Tianjin, 300461, China.
}

\begin{abstract}
Microscopic analysis of soils has attracted wide attention, but at present, the analysis of particle shape of soft clay is still scarce. In this paper, the fractal dimension of particle aspect ratio, roundness and roughness is studied by selecting typical soil samples in Tianjin Port area. The main conclusions are as follows: (1) The average aspect ratio of clay, silty clay and silt is 1.56, 1.58 and 1.65, respectively, which shows that the smaller the soil particles are, the smaller the aspect ratio of the whole soil sample is; (2) The average roundness of clay, silty clay and silt are $0.91,0.89$ and 0.88 , respectively, which indicates that the smaller the soil particles are, the closer the roundness of the whole soil sample is to 1 , the closer the soil particles are to the circle. (3) The smaller the soil particles are, the smoother the surface of the particles is, and the smaller the soil particles are, the more obvious the correlation is.
\end{abstract}

\section{Introduction}

There is a wide range of soft clay in coastal areas of China. Its engineering properties are closely related to the shape, size and gradation of soil particles. According to the previous studies ${ }^{[2-4]}$, the shape characteristics of particles are mainly manifested in three aspects: (1) the elongation property of particles, reflecting the characteristics of the particles as a whole, such as cylinder, rectangle, etc; (2) the number and shape of the boundary of particles, reflecting the number and relationship of edges and edges on the geometrical characteristics of particles; (3) the characteristics of the boundary curve, reflecting the occlusal ability and space filling ability between particles. At present, most of the studies ${ }^{[5-10]}$ focus on the spatial distribution of soil particles and voids or the shape parameters of non-cohesive soils, but the shape analysis of soft clay is not enough.

Table 1. Physical property of test soil samples.
By selecting typical soft clay in Tianjin Port area and using digital image technology, this paper carries out particle shape analysis experiments, and carries out statistical analysis of parameters such as area, circumference, diameter, aspect ratio, roundness and fractal dimension of soil particles, so as to accumulate experience for micro-analysis of clay.

\section{Material and Methods}

\subsection{Test material}

According to the general classification criteria of soil samples, the three soil samples can be roughly divided into three categories: clay, silty clay and silt. The specific physical properties of the three soil samples are shown in Table 1.

\begin{tabular}{cccccc}
\hline soil samples & Water content $\%$ & $\begin{array}{c}\text { Wet density } \\
\mathrm{g} / \mathrm{cm}^{3}\end{array}$ & $\begin{array}{c}\text { liquid limit } \\
\%\end{array}$ & $\begin{array}{c}\text { plasti climit } \\
\%\end{array}$ & plasticity index \\
\hline clay & 43.1 & 1.77 & 46.7 & 22.1 & 24.6 \\
silty clay & 34.9 & 1.84 & 35.1 & 18.8 & 16.3 \\
silt & 25.0 & 1.95 & 20.2 & 14.5 & 5.7 \\
\hline
\end{tabular}

\subsection{Test methods}

The laser image granularity analyzer manufactured by a domestic company is used in the experiment. Its equipment is mainly composed of automatic circulation and dispersion system, Led light source, optical microscope, CCD camera and computer acquisition system. It can recognize and strengthen the edge of particles automatically. It is easy to operate and has good repeatability.

In the experiment, pure water was used as the medium and $4 \%$ sodium hexametaphosphate was added as the dispersant to dilute the soil sample into slurry, open the ultrasonic dispersion system, and then make the soil particles form a stable particle flow through the circulating system. When the soil particles pass through the observation window, the Led beam irradiates the soil particles and makes them optical display. The micro-mirror magnifies the small particles to be measured and imagines them on the photosensitive surface of the CCD camera. The CCD camera converts the optical signals into digital signals and transmits them to the computer system. After the computer receives

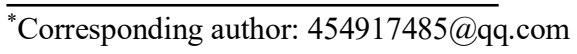


the digital micro-image signals, the image is enhanced and then transformed into a black-and-white binary image, and the particles are extracted. At last, the perimeter (number of pixels), area (number of pixels), long axis and short axis (number of pixels) of the projection of the particle contour are calculated by the system calculation software, and the perimeter, area, length diameter, short diameter, aspect ratio, roundness and equivalent diameter of the particle are calculated according to the magnification factor of the instrument.

\subsection{Particle shape parameters}

At present, there is no uniform standard for the definition of particle shape parameters. In engineering, people usually use globular, polygonal, fibrous, granular, needle-flake, granular and irregular terms to describe ${ }^{[11]}$, but this description is basically qualitative and difficult to carry out quantitative analysis. Therefore, through the comprehensive analysis of the previous research results of particle shape analysis, the following specific shape parameters are defined in order to extract the shape information of particles for quantitative description.

\subsubsection{Aspect ratio}

Aspect ratio is the ratio of the maximum diameter to the minimum diameter of the projection of particles. As shown in Equation (1), this parameter can reflect the elongation property of particles, that is, the degree of the particles as a whole approaching the circle, strip and square. Aspect ratio can roughly describe the shape of particles, which has been widely used in previous studies. The closer a particle is to a square or a circle, the closer its value is to 1 , and the longer the particle is, the larger its value is ${ }^{[12]}$.

$$
\alpha=\frac{L}{B}
$$

In the Equation: $\alpha$ is the aspect ratio; $L$ is the maximum diameter of particle projection; $B$ is theminimum diameter of projection of particles.

\subsubsection{Roundness}

Roundness is the ratio of the equivalent area circumference of particle projection to the actual circumference of particle projection. As shown in Equation (2), this parameter can describe the morphological characteristics of particles as a whole. The closer the general particles are to the standard circle, the closer their values are to 1 , the more the particles deviate from the circle or the larger the fluctuation of the boundary contour (including prominent edges). The smaller the angle, the smaller the value ${ }^{[13]}$.

$$
\theta=\frac{2 \sqrt{\pi A}}{P}
$$

In the Equation: $\theta$ is the roundness; $A$ is the equivalent area of particles; $P$ is the perimeter of boundary contour of particle projection.

\subsubsection{Fractal dimension of roughness}

Fractal theory ${ }^{[14]}$ is a powerful mathematical tool for describing irregular figures and chaotic phenomena in nature. Since it was introduced into geotechnical engineering, a series of research results have been achieved ${ }^{[15-17]}$. In the aspect of particle shape analysis, fractal theory is considered to be an important method to quantitatively describe the complexity of particle contour. Its fractal dimension represents the degree of irregularity or roughness of particle contour. The larger the fractal dimension, the rougher the particle surface. At present, the commonly used methods to obtain fractal dimension are mainly divided into two kinds: fixed-size method and variable-size method. Fixed-size method can be divided into perimeter-maximum diameter method, perimeter-area method and box counting method. In this paper, the fractal dimension of particle surface roughness is obtained by perimeter-area method. For geometric figures with irregular shapes, according to fractal theory, there is a relationship between their perimeter and area, such as Equation (3).

$$
\mathrm{c}=\frac{P^{1 / D_{R}}}{A^{1 / 2}}
$$

In the Equation: $c$ is the constant; $A$ is the equivalent area of particles; $P$ is the perimeter of boundary contour of particle projection; $D_{R}$ is the fractal dimension.

By taking logarithms on both sides of Equation (3), we can get:

$$
D_{R}=2 k
$$

In the Equation: $k$ is the slope of $\lg l-\lg A$.

\section{Results}

\subsection{Aspect ratio}

Figure. 1 shows the statistical results of the aspect ratio of three soil samples. Table 2 further gives the percentage of each aspect ratio interval of soil samples. It can be seen from the charts that the aspect ratio of typical clay in Tianjin Port area distributes from 1 to 7 due to the complex sedimentary environment and particle composition. However, the aspect ratio mainly concentrates between 1 and 2 , and tends to concentrate to 1-2 with the increase of fine particle content in soil samples. Among them, the proportion of clay particles with aspect ratio between 1 and 2 is the largest, followed by silty clay and silt. The average aspect ratios of clay, silty clay and silt are 1.56, 1.58 and 1.65, respectively. This indicates that with the increase of fine particle content in soil samples, the overall aspect ratio of soil particles decreases gradually, the slender particles decrease gradually, and the soil particles tend to be regular. 


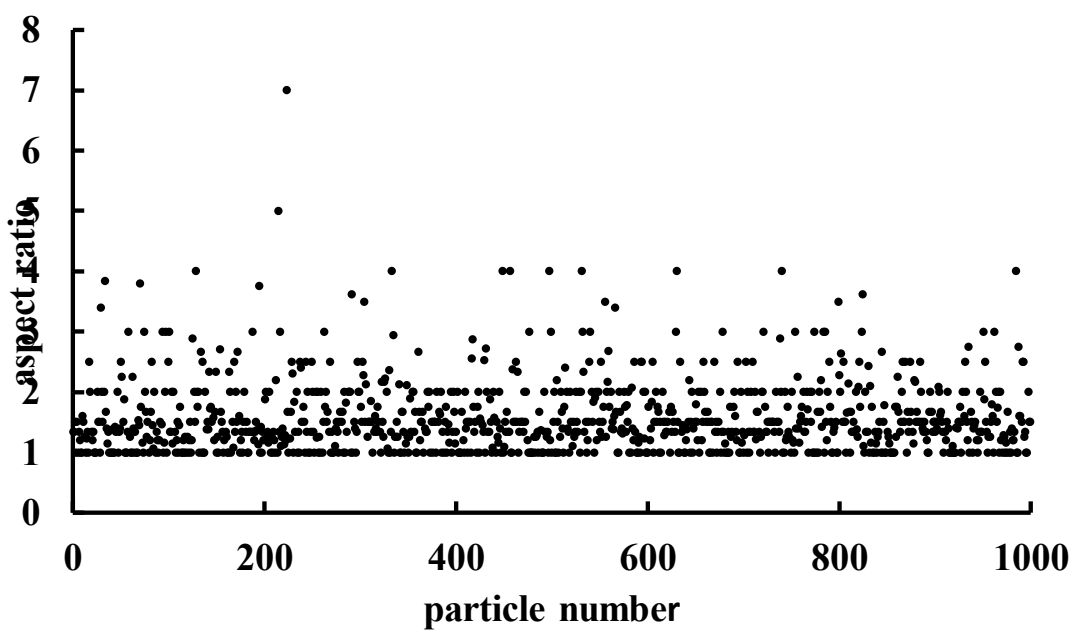

(1) Clay

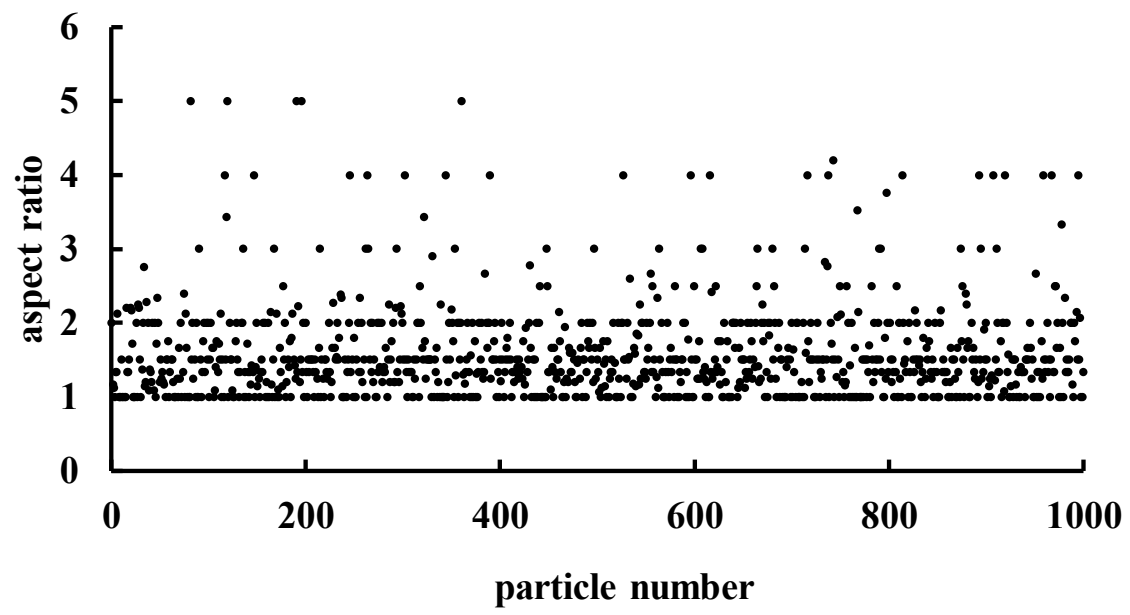

(2) silty clay

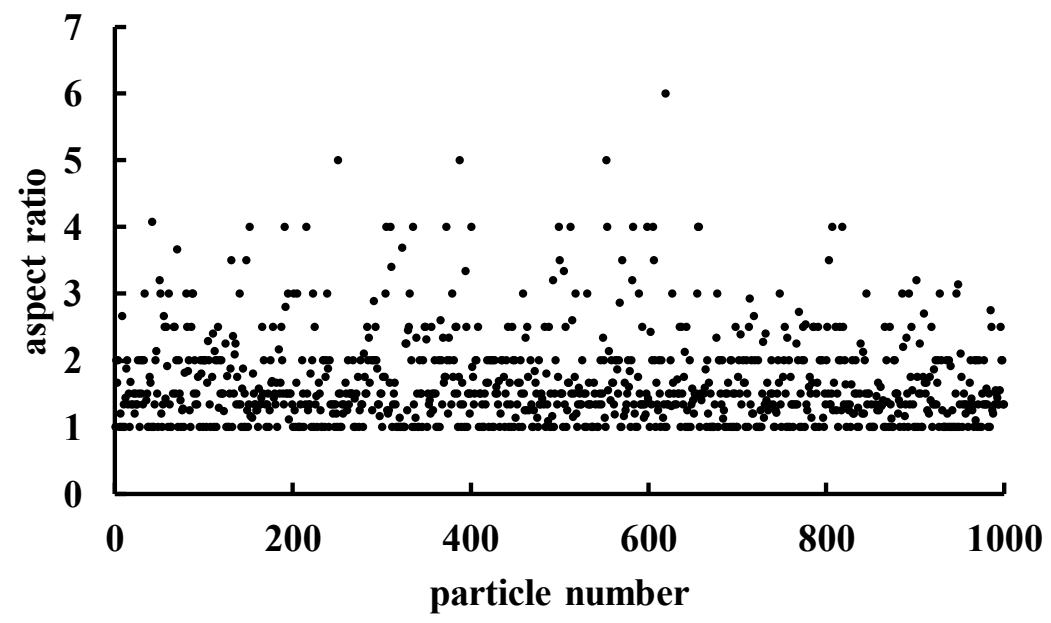

(3) Silt

Figure.1 Statistical result of long-short diameter ratio of test soil samples.

Table 2 Percentage of each aspect ratio interval of test soil sample.

\begin{tabular}{cccccc}
\hline \multirow{2}{*}{ soil samples } & \multicolumn{5}{c}{ percentage of each aspect ratio interval $(\%)$} \\
\cline { 2 - 6 } & $1-2$ & $2-3$ & $3-4$ & $4-5$ & $>5$ \\
\hline clay & 76.7 & 19.0 & 3.2 & 1.0 & 0.1 \\
silty clay & 73.9 & 21.2 & 2.4 & 2.0 & 0.5 \\
silt & 71.6 & 22.1 & 4.1 & 1.8 & 0.5 \\
\hline
\end{tabular}




\subsection{Roundness}

Figure 2 shows the statistical results of the roundness of three soil samples. Table 3 further gives the percentage of each roundness interval of soil samples. From the chart, it can be seen that the circularity of soil samples ranges from 0.4 to 1 , mainly concentrated in $0.9-1$, and with the increase of fine particles in soil samples, the circularity is more concentrated in the range of $0.9-1$. The average roundness of clay, silty clay and silt are $0.91,0.89$ and 0.88 respectively, which indicates that the finer the particles are, the greater the overall roundness of the soil sample is, and the more the shape of the soil particles tends to be round.

Table 3 Percentage of each roundness interval of test soil sample.

\begin{tabular}{cccccc}
\hline \multirow{2}{*}{ soil samples } & \multicolumn{5}{c}{ Percentage of each roundness interval $(\%)$} \\
\cline { 2 - 6 } & $1-0.9$ & $0.9-0.8$ & $0.8-0.7$ & $0.7-0.6$ & $<0.6$ \\
\hline clay & 64.2 & 22.5 & 10.5 & 2.1 & 0.6 \\
silty clay & 62.9 & 21.7 & 12.0 & 2.4 & 1.0 \\
silt & 60.4 & 24.7 & 11.0 & 2.6 & 1.3 \\
\hline
\end{tabular}
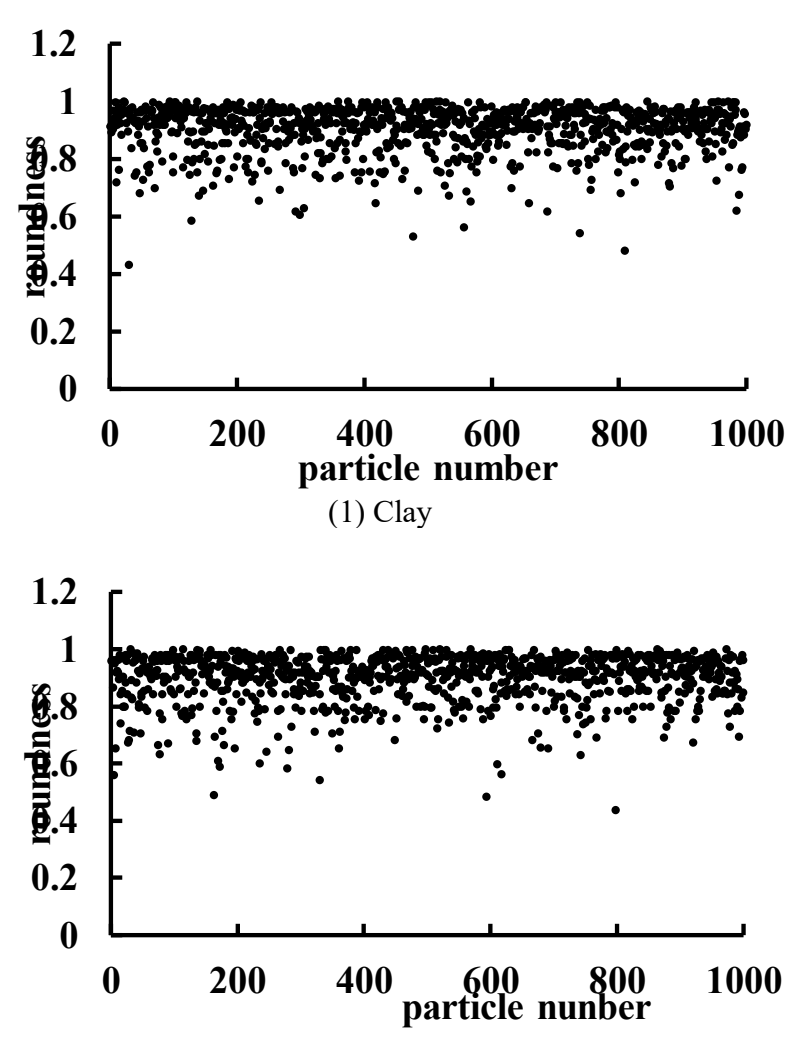

(2) silty clay

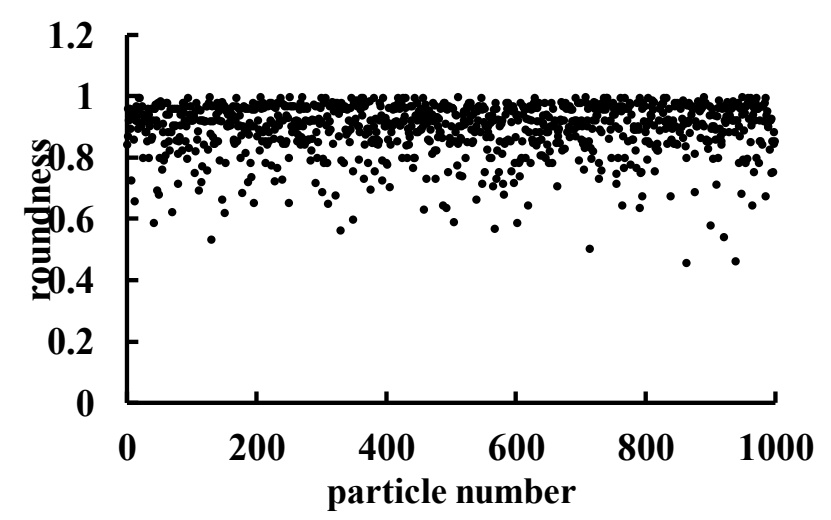

(3) Silt

Figure.2 Statistical result of roundness of test soil samples. 


\subsection{Fractal dimension of roughness}

According to the fractal theory of particle surface roughness, the logarithmic relationship between the perimeter and area of each soil sample is given in Fig. 3, and the fractal dimension statistics of each soil sample roughness is given in Table 4. It can be seen from the chart that the typical clay in Tianjin Port area has good fractal characteristics. The fractal dimensions of surface roughness of clay, silty clay and silt are $0.9590,0.9574$ and 0.9654 , respectively. This shows that the finer the particles are, the smaller the surface fluctuation is, that is, the smoother the surface is, the closer the particle shape is to the circle. At the same time, the logarithmic correlation coefficients of the perimeter and area of clay, silty clay and silt are $0.9176,0.8912$ and 0.8660 , respectively, which indicates that the finer the particles are, the more obvious the correlation is, and the more obvious the fractal characteristics of the particles are. It should be pointed out that the fractal dimension of particle surface roughness obtained by perimeter-area method has only statistical fractal dimension, which reflects the statistical self-similarity between particles and is different from the strict self-similarity of particles themselves.

Table 4 Fractal dimension of surface roughness of soil particles.

\begin{tabular}{cccc}
\hline \multirow{2}{*}{ soil samples } & slope & Fractal dimension & correlation coefficient \\
& $k$ & $D_{R}$ & $R^{2}$ \\
\hline clay & 0.4795 & 0.9590 & 0.9176 \\
silty clay & 0.4787 & 0.9574 & 0.8912 \\
silt & 0.4827 & 0.9654 & 0.8660 \\
\hline
\end{tabular}

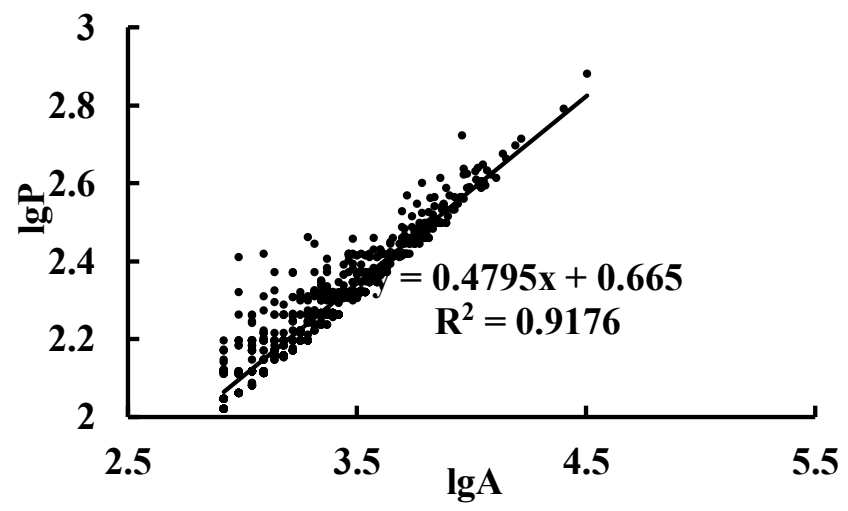

(1) Clay

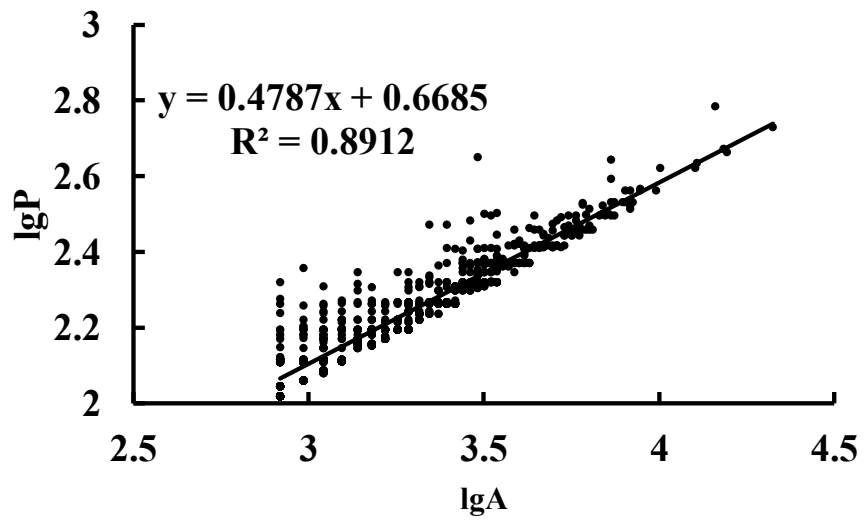

(2) Silty clay 


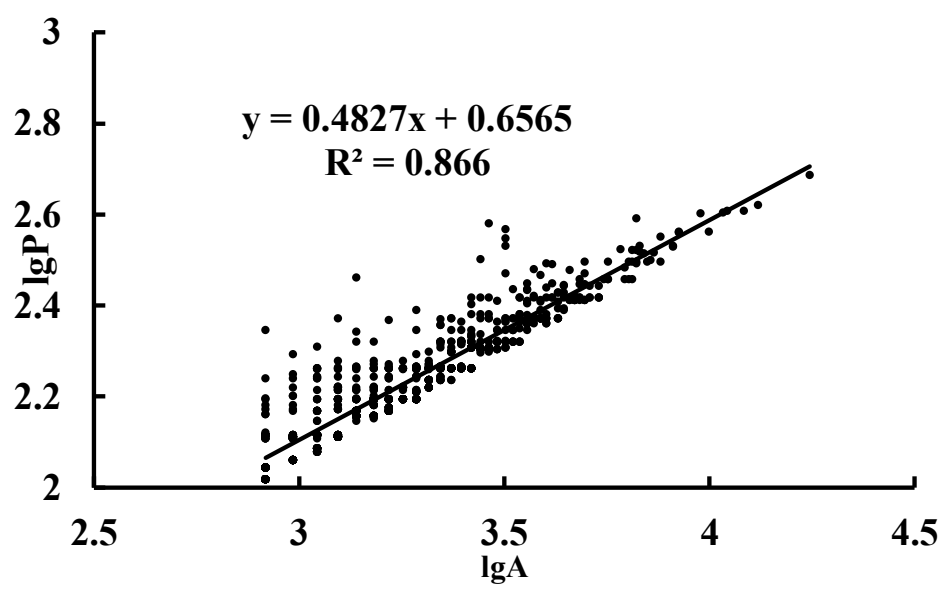

(3) Silt

Figure.3 Logarithmic relationship between perimeter and area of soil particles.

\section{Discussion}

In this paper, the shape of soil particles is observed by digital image technology, and the projection image is obtained. Because the natural soft clay is a three-dimensional particle, the two-dimensional projection will inevitably result in some particles overlapping or joining, which will affect the accuracy of the test results. Therefore, the size, depth and angle of the projection depression area can be identified to determine whether the particles overlap or join. At the same time, when the projection image is quantitatively analyzed, it is necessary to binarize the image and eliminate overlapping or connecting particles, which may cause some distortion of the shape of soil particles.

Most of the typical soft clay in Tianjin Port area is formed by hydraulic transport deposition. Its natural mineral composition is complex. After long-term transport and grinding, most of the particles are finer and rounder, and the finer the particles, the rounder the shape of the particles. From the test results of this paper, it can be seen that the aspect ratio and roundness distribution range of soil particles are larger, and the finer the particles are, the smaller their aspect ratio is, the closer their roundness is to 1 , the smaller the fractal dimension of roughness is, the closer the soil particles are to the circularity, and the smoother the surface of particles is. This is also consistent with the analysis above. The method adopted in this paper is reasonable.

\section{Conclusions}

In this paper, digital image technology is used to carry out particle shape analysis experiments on typical clay in Tianjin Port area of China. The aspect ratio, roundness and roughness fractal dimension of soil samples are analyzed statistically. The main conclusions are as follows:

(1) The aspect ratio of Tianjin Port soil samples mainly concentrates between 1 and 2, and the average aspect ratio of clay, silty clay and silt is $1.56,1.58$ and 1.65 , respectively. This indicates that with the increase of fine particle content in soil samples, the overall aspect ratio of soil particles decreases gradually, the slender particles decrease gradually, and the soil particles tend to be regular shape.

(2) The roundness of soil samples is mainly between
$0.9-1$. The average roundness of clay, silty clay and silt are $0.91,0.89$ and 0.88 , respectively. This shows that the finer the particles are, the larger the overall roundness of soil samples is, and the more the shape of soil particles tends to be rounded.

(3) Soil samples have good fractal characteristics of roughness, and the finer the particles are, the more obvious the fractal characteristics are. The fractal dimensions of surface roughness of clay, silty clay and silt are 0.9590 , 0.9574 and 0.9654 , respectively. This shows that the finer the particles are, the smaller the surface fluctuation is, and the smoother the surface is.

\section{Reference}

1. Shi Bin. Review and Prospect on the microstructure of clayey soil[J]. Journal of Engineering Geology, 1996, (1): 39-44.

2. TU Xinbin, WANG Sijing. Particle shape descriptor in digital image analysis $[\mathrm{J}]$. Chinese Journal of Geotechnical Engineering, 2004, 26(5): 659-662.

3. Zhou Jian, Yu Rongchuan, Jia Mincai. Microstructure Parameters Measurement for Granular Soil Model Using Digital Image Technology[J]. Chinese Journal of Geotechnical Engineering, 2004, 26(5): 659-662.

4. James P Hyslip, Luis E. Vallejo. Fractal analysis of the roughnes sand size distribution of granular materials $[\mathrm{J}]$. Engineering Geology, 1997, 48: 231-243.

5. ALTUHAFI F, O SULLIVAN C, CAVARRETTA I. Analysis of an image-based method to quantify the size and shape of sand particles[J]. Journal of Geotechnical and Geoenvironmental Engineering, 2013, 139(8):1 290-1307.

6. YANG J, LUO X D. Exploring the relationship between critical state and particle shape for granular materials [J]. Journal of the Mechanics and Physics of Solids, 2015, 84:196-213.

7. Kuo C Y, Fost J D, A Chameau J L. Image analysis determination of stereology based fabrictensors [J]. Geotechnique, 1998,48(4):515-525.

8. Lu Haibo, Wang Nian, Zhao Yanlin, et al. Study of structure characteristics evolution of soft clay by pore 
size distribution test $[\mathrm{J}]$. Rock and Soil Manchanics, 2011, 12(S1): 190-197.

9. Jiang Mingjing, Peng Licai, Zhu Hehua, et al. Microscopic investigation on shear band of marine clay in Zhuhai, China[J]. Rock and Soil Manchanics, 2011, 12(S1): 190-197.

10. Lu Haibo, Zhao Yanlin, Kong Lingwei, et al. DETERMINING PARAMETERS OF DAMAGE MODEL OF SOFT SOILS USING MERCURY INTRUSION POROSIMETRY[J]. Journal of Rock Mechanics and Engineering, 2016, 35(4): 768-777.

11. ZENG Fan, HU Yongping, YANG Yi, et al. Particle technology of mineral processing[M]. Xuzhou: China University of Mining and Technology Press, 2001:19-20. (in Chinese)

12. Liu Qingbing, Xiang Wei, M Budhu.Study of particle shape quantification and effect on mechanical property of sand[J]. Rock and Soil Manchanics, 2011, 12(S1): 190-197.

13. Wu Shangwei, Yang Chunhe, Zhang Chao.Microscopic geometric characteristics of surface sedimentary tailings
[J]. Journal of Rock Mechanics and Engineering, 2016, 35(4): 768-777.

14. Mandelbort B B. The Fractal Geometry of Nature[M]. New York: [s.n.]. 1982.

15. TIAN Kan-liang, ZHANG Hui-li, ZHANG Bo-ping, et al. Fractal structure of granularity distribution of natural sedimentary sandy gravel[J]. Journal of Northwest Science Technology University of Agriculture and Forest, 2002, 30(5): 85-89.

16. Xie Heping.Fractal theory-Introduction to Rock Mechanics[M]. Beijing: The Science Publishing Company, 1997.

17. CHEN Jiang-feng, WANG Zhen-fen, YAN Chun-zhong. Fractal description of roundness of clastic particles[J]. Coal Geology \& Exploration, 2002, 30(4): 16-17.

18. Chen Haiyang, Wang Nian, Li Jianguo.Grain shape analysis of calcareous soil[J]. Rock and Soil Manchanics, 2005, 26(9):1389-1392 